\title{
Optimal Environmental Policy and the Dynamic Property in LDCs
}

\author{
MASAHIRO YABUTA \\ Faculty of Economics, Chuo University, 742-1, Higashi-Nakans, Hachioji, Tokyo, Japan
}

(Revised 14 April 2001)

\begin{abstract}
This paper has provided a model framework of foreign assistance policy in the context of dynamic optimal control and investigated the environmental policies in LDCs that received some financial support from abroad. The model framework features a specific behavior of the social planner who determines the level of voluntary expenditure for preservation of natural environment. Because more financial needs for natural environmental protection means less allowance of growth-oriented investment, the social planner confronts a trade-off problem between economic growth and environmental preservation. To tackle with this clearly, we have built a dynamic model with two control variables: per-capita consumption and voluntary expenditure for natural environment.

It has been proved that people in LDCs need a voluntary development of the eco-consciousness for better environmental situation. Because this leads to declining of the rate of economic growth, it may be justified that more foreign aid for domestic economic growth should be financed. By investigating some stability properties of the dynamic system, we have also reached an interesting proposition that the feasibility and effectiveness of the optimal control policy pursued by the social planner of LDCs depend on the people's consciousness towards natural environment.
\end{abstract}

Keywords: Environmental preservation; Foreign aid; LDCs; Common pool resources; Dynamic optimization

JEL Classification: Q28; Q28

\section{INTRODUCTION}

One of major topics in economics is to provide the adequate policy measures for the preservation of natural environment. For the most countries, it is very hard for the social planners to tackle with the environmental problem because they should re-allocate the scarce financial resources towards environmental preservation at the cost of diminishing economic growth.* However, it may be more serious for LDCs since their financial endowments are limited to compare with advanced economies. This accounts for existing international arrangements for foreign assistance from advanced countries to LDCs, leading to a motivation to study the property and effectiveness of such a foreign aid policy. In this paper, we shall investigate how contributions stemmed from the foreign aid work both for protecting natural environment and for raising income level.
Many economists have debated about possible linkages between economic growth and the environmental preservation (see, for example, Kageson (1998) although it is mainly related to non-renewable resources). Economic growth and the environmental preservation still remain to be a complicated issue. In this paper, we shall feature a dynamic model for the optimization policy of LDCs that receives the financial and technological support directly from the advanced countries or via the international organizations. The model type of dynamic optimization employed here is a revised and complex version motivated by many papers including Huang and Cai (1994), van del Ploeg and Lighthard (1994), Rauscher (1994), Dasgupta and Mäler (1995), Mohtadi (1996) Smulders (1998) and Wirl (1999a), for examples. Firstly we shall study a comparative statics to clarify how the foreign aid works for preserving natural environment and what kind of policy mix can be effective when there is

\footnotetext{
*As Kageson (1998, esp. Chapter 17) has pointed out, it is still hard question how much the environmental difficulties are caused by fast growth. Moreover, it is also hard to find evidences how environmental protection costs like pollution abatement costs have a tendency to reduce economic growth.
} 
trade-off between economic growth and the environment. Secondly, we shall mention to the stability properties of the dynamic system. Because our system has two state variables, we shall incorporate a technical procedure into a text to discriminate the stability properties according to the Dockner's method provided by Dockner (1985). $\dagger$

The next section provides the basic model framework with regeneration function of the natural environment. "An optimal environmental policy" section gives the optimal environmental policy and necessary conditions for dynamic optimality. After defining the long-run equilibrium conditions, "The equilibrium" section investigates the effects of changing policy parameters on the equilibrium environment as well as on the voluntary policy to contribute for preserving natural environment. "Dynamic property" section analyzes the stability properties of the dynamic system and investigates the linkages between the stability properties and the policy instruments. Finally, "Concluding remarks" section gives the summary of the paper.

\section{BASIC MODEL}

To clarify the aim of our analysis, our concentration is mainly focused on the economy of LDCs that receives the financial and technological aid from abroad. $\neq$ Because LDCs usually do not have enough financial capacity to facilitate economic program for promoting the economic growth as well as for implementing the natural environmental preservation, it is very conventional for these countries to have some financial support either from a specific advanced country or from the authorities internationally organized like the GEF (global environmental facilities) founded by the World Bank and the UN (See for example, Yabuta (2000)).

In some cases, it is hard to discriminate the effects of spending these environmental facilities on environmental preservation from the induced economic growth effects. Even if the plans are for protecting the tropical rain forest, a forestation can lead to higher revenue of the forestry. However the converse logic is not always true. In fact, so many projects just for economic growth have been executed in LDCs, leading to miserable deterioration of the natural environment. Hence it would be stressed that in LDCs, the social planners must properly assign the financial resources stemmed from the foreign aid to the preservation plans for natural environment.

We shall employ a very simple but usual format-type of production function, which is named the $A K$-type. This is given by

$$
Y=A K,
$$

where $Y, A$ and $K$ represent the output level, a fixed level of capital productivity and the stock of capital, respectively (As for this type of setting, see Barro and Sala-i-Martin (1995) and Hettich (2000)). The market clearing condition is given by

$$
Y=\dot{K}_{1}+C+\delta K+Z_{1}
$$

The r.h.s. of Eq. (2) is the gross domestic expenditure, composed by domestic investment $\left(\dot{K}_{1}\right)$, consumption $(C)$, depreciation of capital $(\delta K$, where $\delta$ is a positive depreciation rate) and government expenditure for protecting the natural environment $\left(Z_{1}\right)$. It is assumed that there is no other government expenditure but for the environmental preservation. It should be noted that we also assume the population of this country to be constant so that all the macro-related variables should be the ones in per capita term.

As far as the foreign aid $(F)$ is concerned, it is assumed that as the international authority or the government of advanced country plans the international or foreign aid program it determines the magnitude of aid mainly based on the size of economic activity of each LDCs. Hence it is natural to have

$$
F=\beta Y,
$$

where $\beta$ is a positive fixed rate of the foreign aid to the income-based activity level of the related economy and it can be a policy instrument mainly controlled by the authorities of the foreign assistance. We shall also incorporate the following equations into our basic model framework.

$$
\dot{K}_{2}=(1-\gamma) \dot{K}_{2}+Z_{2}, \quad Z_{2}=\gamma F
$$

The Eqs. (4) and (5) together imply that the foreign aid is outlaid for two ways; $1-\gamma \%$ of the total foreign aid for the domestic capital formation $\left(\dot{K}_{2}\right)$ and the remains for the environmental preservation $\left(Z_{2}\right)$. Using Eqs. (1)-(3) and (5), the total capital accumulation of the related country is given by

$$
\begin{aligned}
\dot{K} & =\dot{K}_{1}+\dot{K}_{2} \\
& =[\{1+\beta(1-\gamma)\} A-\delta] K-C-Z_{1} .
\end{aligned}
$$

Moreover some simple manipulations lead us to the total expenditure related to the environmental preservation, given by

$$
\begin{aligned}
Z & =Z_{1}+Z_{2}=Z_{1}+\gamma \beta A K=\kappa A K+\gamma \beta A K \\
& =(\kappa+\gamma \beta) A K
\end{aligned}
$$

where $\kappa\left(=Z_{1} / Y\right)$, the ratio of domestic preservation to

\footnotetext{
Wirl (1999a, b) are good examples of applicability of Dockner's method for the dynamic system.

${ }^{\ddagger}$ In this paper we do not mention to the international trade among countries. In this connection, see Rauscher (1994) and Barbier \& Rausher (1994). Although our model do not include the foreign investment explicitly, it is easy to introduce this into analysis because the model is composed as an open system. We also do not mention to the spillover effects of the investment among countries. As for the spillover effects, see Ploeg and Lighthart (1994).
} 
output, represents the index of voluntary contribution to the self-enforced domestic environmental protection plans in terms of economic activity level (see, for example, Beladi et al (1999)).

In the model economy, we are thinking of LDCs where the social planner pursues the rapid economic growth without deteriorating the natural environment. To accomplish this purpose, the social planner must control some policy variables including the spending from foreign aid towards preserving its natural environment. Because an additional unit of spending for the environmental preservation can actually lead to a conservation of the natural environment, it is very natural to feature an environmental preservation function given by

$$
\begin{aligned}
& G=G(Z / K)=G((\kappa+\gamma \beta) A), \quad G^{\prime}>0, \\
& G^{\prime \prime}<0,
\end{aligned}
$$

where $G$ shows the index of effectiveness of the spending, in terms of natural environmental stock, on preserving the natural environment through investment for the ecotechnology or greening development.

On the other side, it is also easy to imagine that if the government does not support a sufficient expenditure to the environmental preservation, the natural environment may be deteriorated. Hence it can be justified that the natural environment is harmfully deteriorated as the spending for natural preservation decreases, leading to

$$
D=D(\alpha Z / K), \quad D^{\prime}<0, \quad D^{\prime \prime}>0
$$

where $D$ shows the index of the damage control. The Eq. (9) shows that the greater the relative size of total spending for environmental protection with respect to capital stock is, the damage control can be more successively effective. Putting Eqs. (8) and (9) together into consideration, we reach a reproduction function of the natural environment, shown as

$$
\begin{aligned}
\dot{R} & =H(R)-D(\alpha Z / K) R+G(Z / K) R \\
& =H(R)-[D(\alpha(\kappa+\gamma \beta) A)-G((\kappa+\gamma \beta) A)] R .
\end{aligned}
$$

The first term of the r.h.s of Eq. (10), that is $H(R)$, shows the natural rate of regeneration of the natural environment while the second and the third term together show the net damage to regenerate through the economic activities in LDCs. "It must be justified to assume that in the process of economic activity, people in LDCs tend to provide the negative effects on natural environment so that the net damage defined by $D-G$ is always positive. ${ }^{\S}$

\section{AN OPTIMAL ENVIRONMENTAL POLICY}

It can be true that the patterns of optimal policy depend on how the social planner of the country takes its policy objectives. We shall here employ the simplest formula of the social welfare function given by the following quasilinear utility function:

$$
U=\log C+\eta R
$$

In Eq. (11), it is clear that social welfare depends positively on the per capita consumption as well as on the natural environment. People in this country assign a weight $\eta$ to the natural environment. An increase in use of natural resources leads to more private consumption and thus a higher level of economic welfare. However it must have an adverse effect on future social welfare because the stock of the natural resources decreases and the quality of natural environment deteriorates.

The objective function to be maximized by the social planner in the country and the related conditions are

$$
\int_{0}^{\infty} U(C, R) \mathrm{e}^{-\rho t} \mathrm{~d} t \rightarrow \max \{C, \kappa\}
$$

subject to Eqs. (6) and (10) and $K(0)=K_{0}$ and $R(0)=R_{0}$ and $0 \leqq \kappa \leqq 1$ where $\rho$ is a positive social discount rate. The optimal policy follows from maximization of inter-temporal social welfare with respect to $C$ as well as $\kappa$."

The current value Hamiltonian $H$ is

$$
\begin{aligned}
H= & {[\log C+\eta R]+\lambda[[(1+\beta(1-\gamma)-\kappa) A} \\
& -\delta] K-C]+\phi[H(R)-\{D(\alpha(\kappa+\gamma \beta) A) \\
& -G((\kappa+\gamma \beta) A)\} R]
\end{aligned}
$$

where the co-state variables $\lambda$ and $\phi$ are the shadow prices of the man-made capital stock and the social value of the natural environmental stock, respectively.

Necessary conditions for optimality are:

$$
\begin{aligned}
\partial H / \partial C & =1 / C-\lambda=0, \partial^{2} H / \partial C^{2}=-1 / C^{2}<0, \\
\partial H / \partial \kappa & =-\lambda A K+\phi\left[G^{\prime}-\alpha D^{\prime}\right] A R=0, \partial^{2} H / \partial \kappa^{2} \\
& =\phi\left[G^{\prime \prime}-\alpha D^{\prime \prime}\right] A^{2} R<0 .
\end{aligned}
$$

The development of the capital stock and the natural environment can be described by the following differential

\footnotetext{
"As for the shape of the regeneration function, $H(R)$, it may be usually exemplified to be a quadratic type of function shown by $H(R)=R\left(R_{\max }-R\right)$, so that $h(R)=H(R) / R$, is linear and $H^{\prime \prime}=-2<h^{\prime}(R)=-1<0$. Hereafter we shall assume such a quadratic type of regeneration function of natural environment.

${ }^{\S}$ This leads us to an additional condition to be presumed. Because $D^{\prime}<0$ and $G^{\prime}>0$, there must be some $(Z / K)_{\max }$ above which $D-G$ becomes negative. We assume that $(Z / K)_{\max }$ is sufficiently large that $D-G$ is accepted to be positive.

The choice of the control variables itself depends on the behavior of the social planner in LDCs. It is easy to assume that another set of variables can be chosen politically to pursue a social optimality. For example, the set of $\{C, \gamma\}$ can be a candidate for the control variables. As far as this case is concerned, it is easy to show that all the conditions for optimality of the system are identical with those given by Eqs. (14)-(17).
} 
equations:

$$
\begin{aligned}
\dot{\lambda} & =-\partial H / \partial K+\rho \lambda \\
& =\lambda[\rho-\{1+\beta(1-\gamma)-\kappa\} A+\delta], \\
\dot{\phi} & =-\partial H / \partial R+\rho \phi \\
& =\phi\left[\rho-H^{\prime}(R)+(D-G)\right]-\eta,
\end{aligned}
$$

where the transversality conditions associated with $K$ and $R$ are:

$$
\begin{aligned}
& \lim _{t \rightarrow \infty} \lambda(t) \exp (-\rho t)=0, \\
& \lim _{t \rightarrow \infty} \phi(t) \exp (-\rho t)=0 .
\end{aligned}
$$

In relation to Eqs. (18) and (19), the extended transversality conditions are:

$$
\hat{\lambda}<\rho, \text { and } \hat{\phi}<\rho .
$$

Equation (14) implies that the marginal cost due to an incremental use in the man-made capital should be equalized for a marginal benefit borne by an increase in consumption. Optimality of the natural environment also appears in Eq. (15), requiring that the marginal income loss due to a decrease in capital investment induced by an incremental finance towards environmental preservation should equal the marginal social value of the preservation of natural environment.

\section{THE EQUILIBRIUM}

Taking logs of Eq. (14), differentiating it with respect to time and taking Eq. (16) into consideration yield the Keynes-Ramsey rule describing the dynamic path of consumption over time:

$$
\hat{C}=\{1+\beta(1-\gamma)-\kappa\} A-\delta-\rho
$$

A balanced growth path is characterized as a dynamic state where all variables grow at a constant rate. Hence we obtain the following equations:

$$
\begin{gathered}
\hat{K}=\hat{Y}=\hat{C}=g, \\
\hat{R}=\hat{\kappa}=0,
\end{gathered}
$$

where $g$ shows the long-run growth rate.

From (6), we have the equation of capital accumulation

$$
\hat{K}=\{1+\beta(1-\gamma)-\kappa\} A-\delta-C / K .
$$

The Eqs. (21) and (24) together lead to the long-run equilibrium condition:

$$
C / K=\rho .
$$

This is the simplest form of the consumption-capital ratio." Because the long-run growth rate, $g$, can be attained when $\kappa=\kappa^{*}$ is realized, it must be given by the following equation:

$$
g=\left\{1+\beta(1-\gamma)-\kappa^{*}\right\} A-\delta-\rho .
$$

As far as the development of the natural environment is concerned, the following equation can be described in the equilibrium:

$$
\begin{aligned}
\hat{R} & =H(R) / R-[D(\alpha(\kappa+\gamma \beta) A)-G((\kappa+\gamma \beta) A)] \\
& =h(R)-[D(\alpha(\kappa+\gamma \beta) A)-G((\kappa+\gamma \beta) A)] \\
& =0 .
\end{aligned}
$$

The r.h.s of Eq. (27) implies that in the long-run equilibrium, the level of natural environment will be the one where the deterioration of the natural environment through human activities is just offset by the nature's power of recovery so that it can be kept at a certain level of the quality as well as the quantity of natural environment. $^{* * *}$

The optimal control of the voluntary policy related to the domestic environmental protection can be described by

$$
\varepsilon\left(G^{\prime}-\alpha D^{\prime} ; \kappa\right) \hat{\kappa}=-\hat{C}+\hat{K}-\hat{\phi}-\hat{R}, .
$$

where $\varepsilon\left(=\mathrm{d}\left(G^{\prime}-\alpha D^{\prime}\right) \kappa /\left(G^{\prime}-\alpha D^{\prime}\right) \mathrm{d} \kappa<0\right)$ is the elasticity of $\kappa$ with respect to the net betterment of natural environment. Taking the long-run equilibrium condition given by Eqs. (15), (23) and (28) into consideration leads to

$$
\begin{aligned}
\hat{\phi} & =\left[\rho-H^{\prime}(R)+(D-G)\right]-\left(G^{\prime}-\alpha D^{\prime}\right) R \eta C / K \\
& =\left[\rho-H^{\prime}(R)+(D-G)\right]-\left(G^{\prime}-\alpha D^{\prime}\right) R \eta \rho \\
& =0 .
\end{aligned}
$$

It should be notable that by total differentiation using Eq. (27) or (29), we obtain $\mathrm{d} R / \mathrm{d} \kappa>0$, meaning a positive corelationship between the voluntary spending policy for environmental preservation and the stock level of natural environment.

The long-run equilibrium $\left(R^{*}, \kappa^{*}\right)$ can be given by Eqs. (27) and (29) simultaneously. Although the policy system we are investigating here employs only two types of control variables; $C$ and $\kappa$, it can be possible to

\footnotetext{
"In this connection, see Hettich (2000), Chapter 3 for example. In our model, both technology and capital depreciation do not affect on the consumption-capital ratio since the elasticity of intertemporal substitution between current and future consumption is unity.

${ }^{* *}$ From Eq. (29), taking assumptions concerning $D$ and $G$ and Eq. (29) into consideration, it is clear that $\rho>H^{\prime}(R)$ since, $\rho-H^{\prime}(R)=$ $\left(\left(G^{\prime}-\alpha D^{\prime}\right) R \eta \rho\right) /(D-G)>0$. Wirl $\left(1999\right.$ a) has pointed out that the sign of $H^{\prime}$ is important for the stability properties of the dynamic optimally controlled system (see his Proposition 1). Our case is corresponding to the class B and class C in his term. As shown later, the stability conditions in our system can incorporate the sign of $H^{\prime}$ through $\phi$ in Eq. (55) when $\eta$ is given.
} 
incorporate the system some other policy measures as the temporal or one-shot cures. As far as our system is concerned, the comparative static analysis can be employed to indicate what impact the changes of $\gamma, \beta$ as well as $\eta$ may have on the long-run equilibrium.

An increase in $\gamma$ means that a policy planner of LDCs is about to assign more and more financial resources stemmed from foreign aid towards preservation of its natural environmental factor. According to the simultaneous Eqs. (27) and (28), the effects of change in $\gamma$ on $R^{*}$ and $\kappa^{*}$ can be described as

$$
\begin{gathered}
\mathrm{d} R^{*} / \mathrm{d} \gamma=0, \\
\mathrm{~d} \kappa^{*} / \mathrm{d} \gamma=-\beta<0 .
\end{gathered}
$$

In addition, the effect of changes in $\gamma$ on the long-run economic growth rate, $g$ can be given by

$$
\mathrm{d} g / \mathrm{d} \gamma=0 .
$$

Equations (30)-(32) leads us to an interesting proposition, implying that under the optimal control policy given by Eq. (12) followed by the government of LDCs, a policy change in terms of domestic voluntary quota of outlays to natural preservation has no effect on the long-run economic growth rate. In the long-run equilibrium, the policy planner can preserve the level of natural environment from being deteriorated even in the case of decrease in $\gamma$. This is because the positive effects via an increase in domestic investment, which is voluntarily planned for protecting domestic natural environment, should offset the negative effects on natural environment through a decrease in quota from foreign assistance. In so far as the social planner follows the optimal policy rule, a change is in $\gamma$ will not affect the long-run equilibrium.

An increase in $\beta$, the rate of foreign aid to the domestic income of a under developing economy, has the following impacts on the long-run equilibrium conditions given by

$$
\begin{gathered}
\mathrm{d} R^{*} / \mathrm{d} \beta=0, \\
\mathrm{~d} \kappa^{*} / \mathrm{d} \beta=-\gamma<0,
\end{gathered}
$$

and

$$
\mathrm{d} g / \mathrm{d} \beta=1-\gamma-\mathrm{d} \kappa / \mathrm{d} \beta=1>0 .
$$

Equation (33) shows that a change in $\beta$, just like a change in $\gamma$, does not affect the level of natural environment. Equation (34) implies that for a given level of $\gamma$, an increase in $\beta$ leads to a decrease in the level of voluntary expenditure for preserving natural environment, $\kappa$. This is because a higher level of $\beta$ will give a sufficient volume of foreign financial aid by which the social planner can easily afford to preserve the natural environment even if $\kappa$ is reduced. In contrast to the case of $\gamma$, an increase in $\beta$ will cause the long-run growth rate to increase.

Changes in $\eta$ mean a changing pattern of the preference between human-made goods and natural environment in this country. It is very natural to imagine that $\eta$ will increase as people in this country turns to be more environment-friendly and eco-aware. A recent movement for global environmental preservation will lead to an increase in $\eta$ via so many channels including the international arrangement for preserving the global environment as well as the environmental education programs provided by international organizations such as UNEP. As far as this country is concerned, it is very likely to occur that people will change the level of $\eta$ through many channels of the foreign assistance programs they take. In our model, if $\eta$ is sufficiently small, then we have $^{\dagger \dagger}$

$$
\begin{aligned}
& \mathrm{d} R^{*} / \mathrm{d} \eta>0, \\
& \mathrm{~d} \kappa^{*} / \mathrm{d} \eta>0 .
\end{aligned}
$$

It is clear, from Eqs. (36) and (37), that a changing pattern towards more environment-friendly behavior of the people in this country, i.e. an increase in $\eta$, can lead to an increase in the domestic voluntary expenditure for preserving the natural environment. This must help the stock level of natural environment to increase. However it should be noted that an increase in $\eta$ has the negative impact on the long-run equilibrium growth rate of the economy:

$$
\mathrm{d} g / \mathrm{d} \eta<0 .
$$

Because it seems to be the case that the social planners of LDCs would like to avoid a decrease in economic growth rate, it must be difficult for them to accept a greater $\eta$. Equation (38) implies a trade-off between economic growth and environmental preservation for LDCs. To improve the natural environmental quality or to increase the stock of natural environment, people need a voluntary development of the preference pattern towards ecoconsciousness. In this case, however, they are forced to have a diminishing economic growth. Hence the following proposition will hold that a contributor of the foreign assistance must employ an adequate level of $\beta$ (increase in $\beta$ ) in the case where people tend to be more environmentfriendly (increasing in $\eta$ ). Therefore it would be justified

${ }^{\dagger} \mathrm{By}$ a comparative static procedure, we have the following equations for $\mathrm{d} R$ and $\mathrm{d} \eta$ :

$$
\left[\begin{array}{cc}
h^{\prime} & -\left(\alpha D^{\prime}-G^{\prime}\right) A \\
-H^{\prime \prime}-\left(G^{\prime}-\alpha D^{\prime}\right) \eta \rho & \left(\alpha D^{\prime}-G^{\prime}\right) A-\left(G^{\prime \prime}-\alpha^{2} D^{\prime \prime}\right) A R \eta \rho
\end{array}\right]\left[\begin{array}{l}
\mathrm{d} R \\
\mathrm{~d} \kappa
\end{array}\right]=\left[\begin{array}{c}
0 \\
\left(G^{\prime}-\alpha D^{\prime}\right) R \rho
\end{array}\right] \mathrm{d} \eta .
$$

Hence it is easy to see that if $\eta=0$, we obtain $\mathrm{d} R / \mathrm{d} \eta=\left(G^{\prime}-\alpha D^{\prime}\right) R \rho /\left(h^{\prime}-H^{\prime \prime}\right)$ and $\mathrm{d} \kappa / \mathrm{d} \eta=-h^{\prime} R \rho\left(h^{\prime}-H^{\prime \prime}\right) A$. As mentioned before about the shape of regeneration function of the natural environment, it may be reasonable to accept $H^{\prime \prime}<h^{\prime}<0$, leading to $\mathrm{d} R / \mathrm{d} \eta>0$ and $\mathrm{d} \kappa / \mathrm{d} \eta>0$ for sufficiently small $\eta$. 
that more foreign aid should be financed when the people of LDCs change their voluntary policy pattern towards environmental preservation.

\section{DYNAMIC PROPERTY}

In this section we shall investigate the stability properties of the dynamic system composed by two state variables, $K$ and $R$ and two co-state variables, $\lambda$ and $\phi$. By using Eqs. (14) and (15), a simple manipulation yields

$$
C=1 / \lambda,
$$

and

$$
\kappa=\kappa(K, R, \lambda, \phi ; \gamma, \beta) .
$$

It should be noted that in Eq. (40), $\kappa$, the preservationoutput ratio, is shown as a reduced form with respect to four endogenous variables, $K, R, \lambda$ and $\phi$ as well as the

$$
\begin{aligned}
& \dot{\lambda}=\lambda[\rho-\{1+\beta(1-\gamma)-\kappa\} A-\delta]=\Omega_{3} \\
\dot{\phi}= & \phi\left[\rho-H^{\prime}(R)+\{D(\alpha(\kappa+\gamma \beta) A)-G((\kappa+\lambda \beta) A)\}\right] \\
& -\eta \\
= & \Omega_{4}
\end{aligned}
$$

It should be noted that in the long-run equilibrium, from Eqs. (16), (22), (23) and (29), we obtain

$$
\hat{K}=-\hat{\lambda}=g, \quad \dot{R}=\dot{\phi}=0 .
$$

These conditions characterize the steady state of the system $S$. To analyze the stability property of the system $S$ by means of local stability analysis, we shall consider the linearized system around the steady state.

The Jacobian matrix of $S$ evaluated at the steady state is (see right-hand column for equation (48).

$$
\begin{aligned}
J & =\left[\begin{array}{cccc}
\Omega_{1 K}-g & \Omega_{1 R} & \Omega_{1 \lambda} & \Omega_{1 \phi} \\
\Omega_{2 K} & \Omega_{2 R} & \Omega_{2 \lambda} & \Omega_{2 \phi} \\
\Omega_{3 K} & \Omega_{3 R} & \Omega_{3 \lambda}+g & \Omega_{3 \phi} \\
\Omega_{4 K} & \Omega_{4 R} & \Omega_{4 \lambda} & \Omega_{4 \phi}
\end{array}\right] \\
& =\left[\begin{array}{cc}
\rho-A K \kappa_{K} & -A K \kappa_{R} \\
-\left(\alpha D^{\prime}-G^{\prime}\right) A R \kappa_{K} & H^{\prime}-(D-G)-\left(\alpha D^{\prime}-G^{\prime}\right) A R \kappa_{R} \\
A \lambda \kappa_{K} & A \lambda \kappa_{R} \\
A \phi\left(\alpha D^{\prime}-G^{\prime}\right) \kappa_{K} & \phi\left[-H^{\prime \prime}+\left(\alpha D^{\prime}-G^{\prime}\right) A \kappa_{R}\right]
\end{array}\right.
\end{aligned}
$$

policy instrumental parameters, $\gamma$ and $\beta$. The signs of partial derivative of Eq. (40) with respect to each variable in r.h.s of Eq. (40) are calculated as follows;

$$
\begin{gathered}
\partial \kappa / \partial K \equiv \kappa_{K}=\lambda /\left[A \phi R\left(G^{\prime \prime}-\alpha^{2} D^{\prime \prime}\right)\right]<0, \\
\partial \kappa / \partial R \equiv \kappa_{R}=-\left(G^{\prime}-\alpha D^{\prime}\right) /\left[A R\left(G^{\prime \prime}-\alpha^{2} D^{\prime \prime}\right]\right. \\
>0 \\
\partial \kappa / \partial \lambda \equiv \kappa_{\lambda}=K /\left[A \phi R\left(G^{\prime \prime}-\alpha^{2} D^{\prime \prime}\right)\right]<0, \\
\partial \kappa / \partial \phi \equiv \kappa_{\phi}=-\left(G^{\prime}-\alpha D^{\prime \prime}\right) /\left[A \phi\left(G^{\prime \prime}-\alpha^{2} D^{\prime \prime}\right)\right] \\
>0, \\
\partial \kappa / \partial \gamma \equiv \kappa_{\gamma}=-\beta<0, \\
\partial \kappa / \partial \beta \equiv \kappa_{\beta}=-\gamma<0 .
\end{gathered}
$$

Then the system can be summed up in the following dynamic system, $S$, for the optimal solutions.

$$
\begin{gathered}
\dot{K}=[\{1+\beta(1-\gamma)-\kappa\} A-\delta] K-1 / \lambda=\Omega_{1} \\
\dot{R}=H(R)-[D(\alpha(\kappa+\gamma \beta) A)-G((\kappa+\gamma \beta) A)] R=\Omega_{2}
\end{gathered}
$$

$$
\left.\begin{array}{cc}
-A K \kappa_{\lambda}+1 / \lambda^{2} & A K \kappa_{\phi} \\
-\left(\alpha D^{\prime}-G^{\prime}\right) A R \kappa_{\lambda} & -\left(\alpha D^{\prime}-G^{\prime}\right) A R \kappa_{\phi} \\
A \lambda \kappa_{\lambda} & A \lambda \kappa_{\phi} \\
A \phi\left(\alpha D^{\prime}-G^{\prime}\right) \kappa_{\lambda} & \rho-H^{\prime}+(D-G)+A \phi\left(\alpha D^{\prime}-G^{\prime}\right) \kappa_{\phi}
\end{array}\right]
$$

The characteristic roots of $J$ must be the solutions of the characteristic equation:

$$
|J-p I|=0
$$

where $I$ is the identity matrix of $4 \times 4$ and $p$ represents the eigenvalues of $J$. The local stability property is determined by the eigenvalues of Eq. (49) obtained by a polynomial of degree four

$$
p^{4}-(\operatorname{tr} J) p^{3}+\left(\Lambda+\rho^{2}\right) p^{2}-\psi p+\operatorname{det} J=0,
$$

where $\Lambda$ and $\psi$ are the sum of the principle minors of $J$ of dimension 2 and 3, respectively. Equation (50) can be solved for four eigenvalues, $p_{\mathrm{i}}, \mathrm{i}=1,2,3,4$.

To accomplish our task here we shall apply a method developed by Dockner (1985) by which the stability properties of the linearized optimal dynamics with fourdimensional state and co-state variables can be investigated in a single way. Following his procedure leads us to the formula related to $J$ :

$$
\operatorname{tr} J=\sum_{i=1}^{4} p_{i}=2 \rho>0
$$




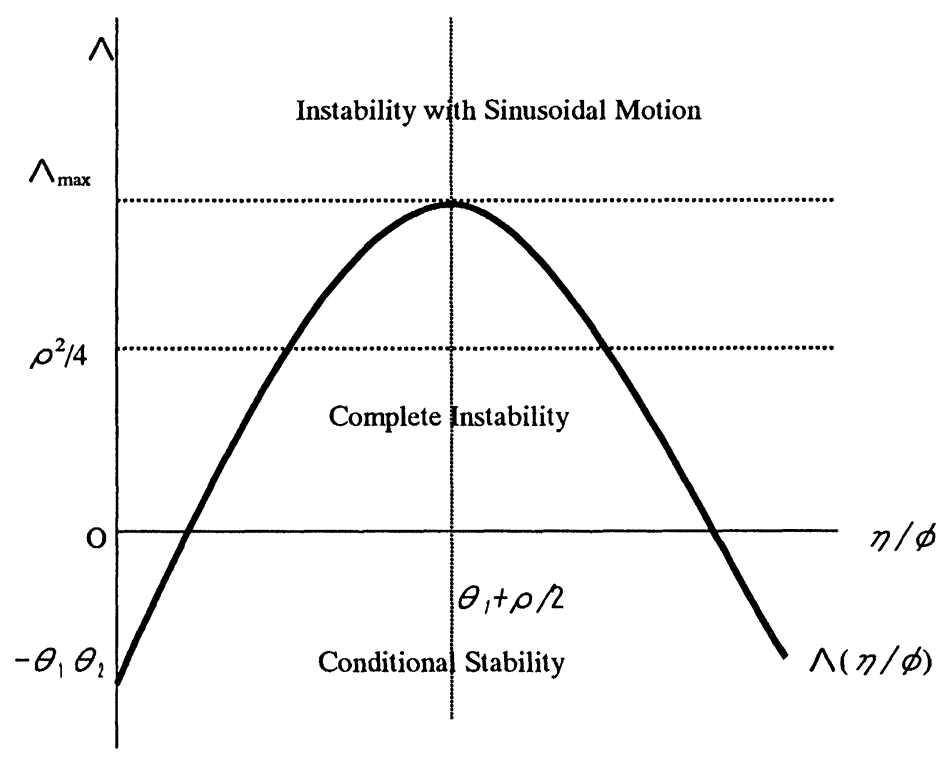

FIGURE 1 Stability property of the system $S$.

and

$$
\operatorname{det} J=\prod_{i=1}^{4} p_{i},
$$

where $\operatorname{tr} J$ and $\operatorname{det} J$, respectively, represent the trace and determinant of $J$. The determinant of $J$ can be explicitly calculated of the form;

$$
\begin{aligned}
\operatorname{det} J= & A \lambda\left(\rho-\frac{\eta}{\phi}\right) \frac{\eta}{\phi} \times[\rho K-1 / \lambda] /\left\{A \phi R \left(G^{\prime \prime}\right.\right. \\
& \left.\left.-\alpha^{2} D^{\prime \prime}\right)\right\}
\end{aligned}
$$

Hence it is easy to find that in the steady state of the system $S$, det $J$ must be zero because $\rho K=C=1 / \lambda$ is assured from Eqs. (25) and (39). As far as $\Lambda$ is concerned, we obtain

$$
\begin{aligned}
\Lambda= & \left|\begin{array}{cc}
\Omega_{1 K}-g & \Omega_{1 \lambda} \\
\Omega_{3 K} & \Omega_{3 \lambda}+g
\end{array}\right|+\left|\begin{array}{cc}
\Omega_{2 R} & \Omega_{2 \phi} \\
\Omega_{4 R} & \Omega_{4 \phi}
\end{array}\right| \\
& +2\left|\begin{array}{ll}
\Omega_{1 R} & \Omega_{1 \phi} \\
\Omega_{3 R} & \Omega_{3 \phi}
\end{array}\right|
\end{aligned}
$$

From Eqs. (48) and (54), $\Lambda$ can be expressed by a quadratic equation of the parameter $\eta$ in an explicit way as follows:

$$
\Lambda=\Lambda(\eta / \phi)=-\left(\frac{\eta}{\phi}\right)^{2}+\left(\rho+2 \theta_{1}\right)\left(\frac{\eta}{\phi}\right)-\theta_{1} \theta_{2},
$$

where

$$
\theta_{1}=\left(\alpha D^{\prime}-G^{\prime}\right)^{2} /\left(\alpha D^{\prime \prime}-G^{\prime \prime}\right)>0,
$$

and

$$
\theta_{2}=\rho-H^{\prime \prime} R>0
$$

In relation to Eq. (55), it is also notable that $\Lambda(0)=$ $-\theta_{1} \theta_{2}<0$ and $\Lambda\left(\theta_{1}+\rho / 2\right)=\Lambda_{\max }$.

Dockner (1985) has also explicitly derived a simple formula for the eigenvalues of the system. Following his procedure we obtain

$$
{ }_{3}^{4} p_{1}^{2}=\frac{\rho}{2} \pm \sqrt{\left(\frac{\rho}{2}\right)^{2}-\frac{\Lambda}{2} \pm \frac{1}{2} \sqrt{\Lambda^{2}-4 \operatorname{det} J}} .
$$

By taking the eigenvalues given in Eq. (58) and $\operatorname{det} J=0$ into consideration, it is easy to reach the following result related to four eigenvalues:

$$
\begin{gathered}
p_{1}=0, \\
p_{2}=\rho>0, \\
p_{3}=\frac{\rho+\sqrt{\rho^{2}-4 \Lambda}}{2}, \\
p_{4}=\frac{\rho-\sqrt{\rho^{2}-4 \Lambda}}{2} .
\end{gathered}
$$

Hence the system $S$ can never be completely stable because $p_{2}>0$. As far as $p_{3}$ and $p_{4}$ are concerned, the results obtained from Eqs. (55), (61) and (62) lead us to the following propositions:

(i) If $\rho^{2} / 4>\Lambda$, then $p_{3}$ as well as $p_{4}$ cannot be the complex number. On the contrary, if $\rho^{2} / 4<\Lambda$, then $p_{3}$ and $p_{4}$ can have the imaginary part.

(ii) One of the following five cases can occur;

(Case 1) $\Lambda<0 \Rightarrow p_{3}>0, p_{4}<0$

(Case 2) $0=\Lambda \Rightarrow p_{3}=\rho>0, p_{4}=0$

(Case 3) $0<\Lambda<\rho^{2} / 4 \Rightarrow p_{3}>0, p_{4}>0$

(Case 4) $\rho^{2} / 4=\Lambda \Rightarrow p_{3}=p_{4}=\rho / 2>0$

\footnotetext{
${ }^{*}$ For the conditions of the stability property, Dockner (1985) has given Theorem 2,3 , and 4 . He has also given a remark for the case of det $J=0$ and
} $\Lambda<0$. This is corresponding to Case 1 where we have one zero, two positive (one equal to $\rho$ ) and one negative eigenvalue. 
(Case 5) $\rho^{2} / 4<\Lambda \Rightarrow p_{3}=\rho+b i, p_{4}=\rho-b i \quad(i$; imaginary number)

From these propositions, it is clear that there is no saddle point case and the system $S$ can be conditional stable only when the condition in Case 1 , negativity of $\Lambda$, is assured. In this case, two eigenvalues are positive, one eigenvalue is negative and one eigenvalue is zero. Although the system $S$ degenerates when $\operatorname{det} J=0$, the optimal controls pursued by the social planner can ensure convergence to the steady state. It must be notable that in all other cases except for Case 1, three eigenvalues of $S$ have a positive real part and one eigenvalue is zero, meaning the case of completely instable. Therefore, as far as the system $S$ is concerned, there may be the case where the social planner cannot control their control variables, $\kappa$ and $R$, so as to make solutions converge towards the steady state.

The propositions imply that the social planner can manipulate the control variables optimally along the admissible paths only when $\Lambda$ is negative. To see the conditions related the negativity of $\Lambda$, we shall take Eq. (55) into consideration. From Eq. (55), we obtain the quadratic relation between $\Lambda$ and $\eta / \phi$ depicted as in Fig. 1 . Figure 1 shows how the instability property of system $S$ depends on the level of $\eta$ for a given $\phi$. Note that the conditional stability can be assured when $\eta$ is sufficiently small or, on the contrary, sufficiently large. As mentioned before, $\eta$ means the level of eco-consciousness of the people. If the people living in LDCs have just started to think of preserving the natural environment, one may suppose that $\eta$ still remains so small. If this is the case in germ of economic development process, the social planner can accomplish its goals for sustainable development along the dynamic optimal control program. Then the contributors had better increase the level of foreign assistance to LDCs. However, if it is not the case, the social planner of this country should not follow the dynamic control policy investigated in this paper. Accordingly it leads to our major conclusion that whether the optimal control policy pursued by the social planner of LDCs is feasible or not is mostly dependent on the people's consciousness towards natural environment.

\section{CONCLUDING REMARKS}

We have extended a new model framework of foreign assistance policy in context of the dynamic optimal control and investigated the economic consequences of foreign aid policy for LDCs that have received some financial support from abroad. The model framework features a specific behavior of the social planner who determines the level of voluntary expenditure for preservation of natural environment. In general, more financial needs for natural environmental protection leads to less allowance of growth-oriented investment. Therefore the social planner is always forced to confront a kind of trade-off between economic growth and the environmental preservation. To incorporate this problem into a model analysis clearly, we have built a dynamic model with two control variables: per-capita consumption and voluntary expenditure for natural environment.

As far as the long-run equilibrium is concerned, we have proved that in order to reach a better situation of natural environment, people in LDCs need a voluntary development of the eco-consciousness. Because this leads to declining of the rate of economic growth, it may be justified that more foreign aid for domestic economic growth should be financed. By investigating some stability properties of the dynamic system, we have also reached an interesting proposition that the feasibility and effectiveness of the optimal control policy pursued by the social planner of LDCs depends on the people's consciousness towards natural environment. This also implies that as far as the eco-consciousness is concerned, a halfway position may be worse for the optimal control policy.

\section{References}

Barbier, E.B. and Rauscher, M. (1994) "Trade, tropical deforestation and policy interventions", In: Carraro, C., ed, Trade, Innovation, Environment (Kluwer Academic Publishers, Dordrecht).

Barro, R.J. and Sala-i-Martin, X. (1995) Economic Growth (McGrawHill, New York)

Beladi, H., Chi-Chur, Chao and Frasca, R. (1999) "Foreign investment and environmental regulations in LDCs", Resource and Energy Economics 21, 191-199.

Dasgupta, P. and Mäler, K.-G. (1995) "Poverty, institutions and the environmental resource-base", In: Behrman, J. and Srinivasan, T.N., eds, Handbook of Development Economics (North-Holland, Amsterdam).

Dockner, E. (1985) "Local stability analysis in optimal control problems with two state variables", Optimal Control and Economic Analysis 2, 89-103.

Hanley, H., Shogren, J.F. and White, B. (1997) Environmental Economics in Theory and Practice (Macmillan Press, New York).

Hettich, F. (2000) Economic Growth and Environmental Policy, A Theoretical Approach (Edward Elgar).

Huang, C.H. and Cai, D. (1994) "Constant-returns endogenous growth with pollution control", Environmental and Resource Economics 4, 383-400.

Kageson, P. (1998) Growth Versus the Environment: Is there a Trade-off? (Kluwer Academic Publishers, Dordrecht).

Kamien, M.I. and Schwartz, N.L. (1991) Dynamic Optimization, 2nd ed. (North-Holland, Amsterdam).

Mohtadi, H. (1996) "Environment, growth and optimal policy design", Journal of Public Economics 63, 119-140.

van der Ploeg, F. and Lighthart, J.E. (1994) "Sustainable growth and renewable resources in the global economy", In: Carraro, C., ed, Trade, Innovation, Environment (Kluwer Academic publishers, Dordrecht), pp 259-280.

Rauscher, M. (1994) "Foreign trade and renewable resources", In: Carraro, C., ed, Trade, Innovation, Environment (Kluwer Academic publishers, Dordrecht), pp 109-121.

Smulders, S. (1998) "Technological change, economic growth and sustainability", In: van den Berg, Jeroen C.J.M. and Hofkes, M.W., eds, Theory and Implementation of Economic Models for Sustainable Development (Kluwer Academic Publishers, Dordrecht), pp 37-65.

Yabuta, M. (2000) "Some aspects on the global environmental policy (in Japanese)", Global Environmental Policy in Japan (Chuo University Press, Tokyo) Vol. 1, 17-24.

Wirl, F. (1999a) “Complex, dynamic environmental policies”, Resource and Energy Economics 21, 19-41.

Wirl, F. (1999b) "De- and reforestation: stability, instability and limit cycles", Environmental and Resource Economics 14, 463-479. 


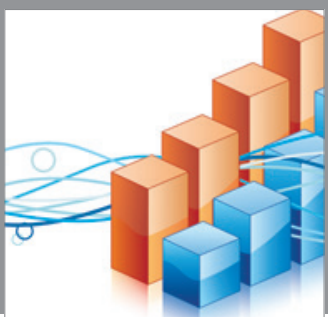

Advances in

Operations Research

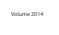

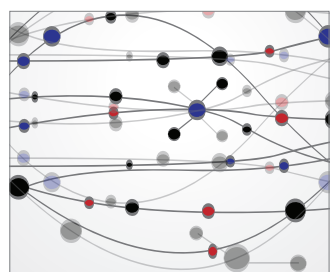

\section{The Scientific} World Journal
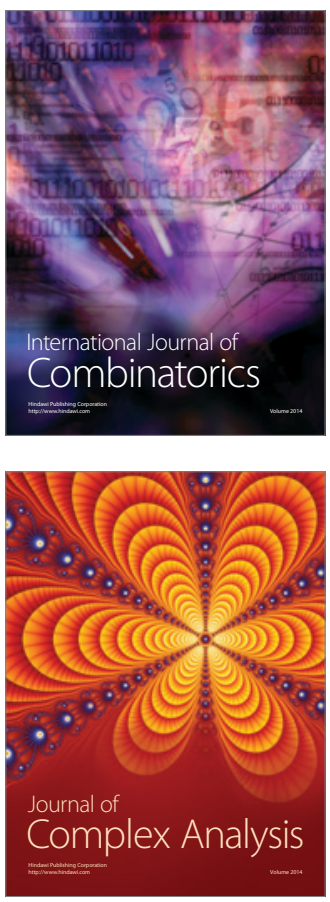

International Journal of

Mathematics and

Mathematical

Sciences
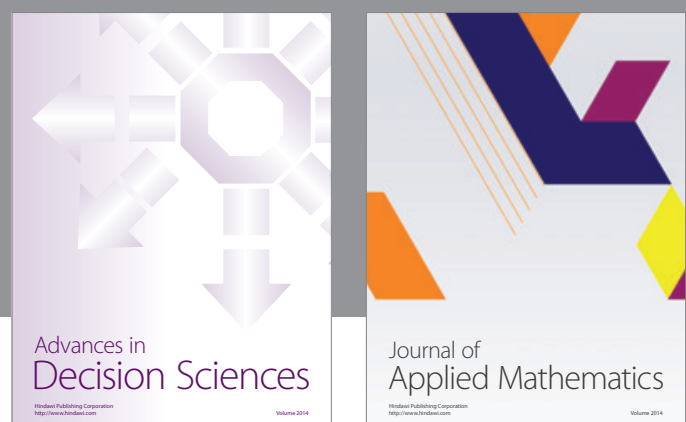

Journal of

Applied Mathematics
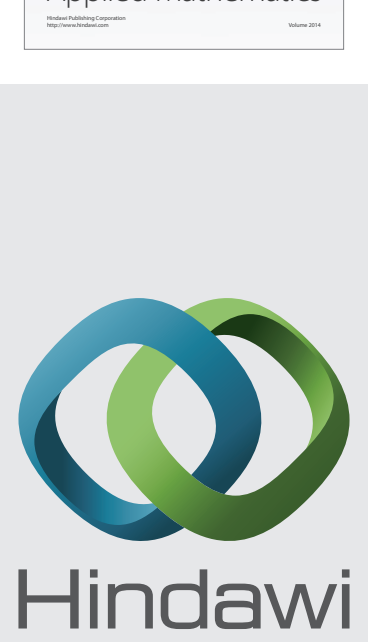

Submit your manuscripts at http://www.hindawi.com
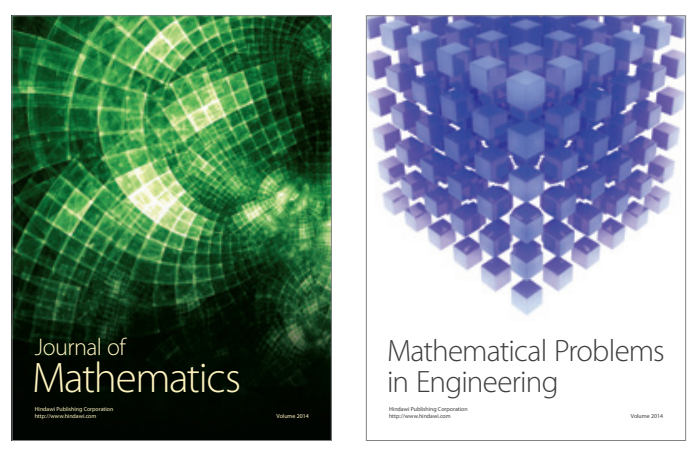

Mathematical Problems in Engineering
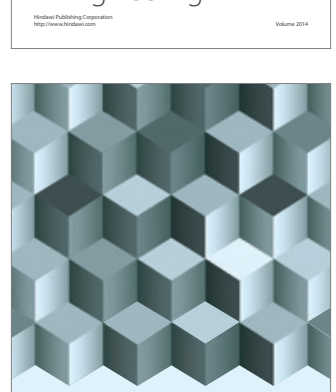

Journal of

Function Spaces
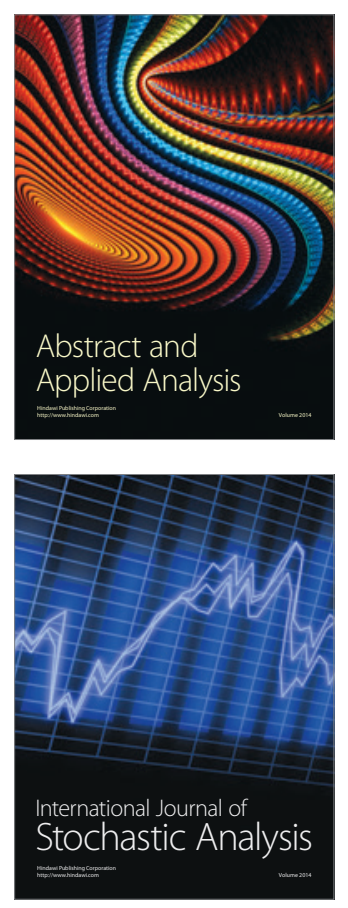

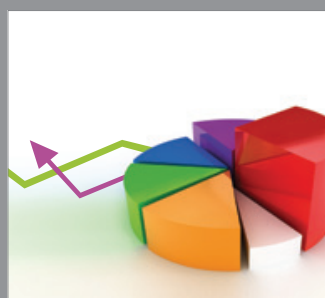

ournal of

Probability and Statistics

Promensencen
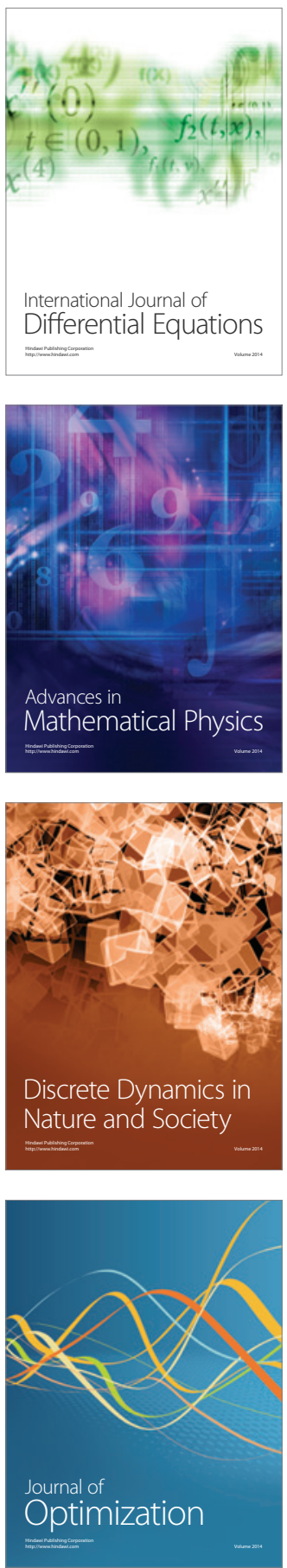